\title{
CAPTURE DANS LE RHIN DE REPRÉSENTANTS DES ESPĖCES ASPIUS ASPIUS (LINNÉ 1758) ET VIMBA VIMBA (LINNÉ 1758)
}

\author{
J.B. SCHWEYER (1), J. ALLARDI (2) et M. DORSON (3)
}

(1) Délégation Régionale No 3 du C.S.P., 18 rue de Nomeny, 57158 MONTIGNY-LES-METZ

(2) CEMAGREF, Division Qualité des Eaux, Pêche et Pisciculture, 14 avenue de SaintMandé, 75012 PARIS

(3) Laboratoire de Virologie et d'Immunologie Moléculaires, Unité Virus des Poissons, INRA, Domaine de Vilvert, 78352 JOUY-EN-JOSAS CEDEX

\section{RÉSUMÉ}

Un cyprinidé identifié comme appartenant à l'espèce Aspius aspius a été capturé en 1988 par pêche électrique dans le Rhin là où celui-ci sert de frontière entre la France et l'Allemagne. En 1989 c'est un autre cyprinidé déterminé comme Vimba vimba qui a été pris à la ligne depuis la rive française du Rhin. Ces deux espèces n'avaient encore pas été signalées en France.

\section{INDIVIDUALS BELONGING TO THE SPECIES ASPIUS ASPIUS (LINNAEUS 1758) AND VIMBA VIMBA (LINNAEUS 1758) CAPTURED IN THE RHINE}

\section{SUMMARY}

A cyprinid fish identified as belonging to the species Aspius aspius was captured by electro-fishing in the Rhine where this river separates France from Germany. In 1989 an other cyprinid was caught by an angler from the french bank of the Rhine and determined as Vimba vimba. These two species had not been recorded before in France.

\section{ORIGINE DES POISSONS}

Des individus appartenant à deux espèces de cyprinidés qui ne faisaient pas partie jusqu'à présent de la faune de France ont été récemment capturés dans le Rhin depuis la rive française. Ils ont été identifiés comme représentants des deux espèces suivantes :

$$
\begin{aligned}
& \text { Aspius aspius (Linné 1758) } \\
& \text { Vimba vimba (Linné 1758) }
\end{aligned}
$$

Le représentant de la première espèce (l'Aspe) a été capturé par pêche électrique le 29/10/88 à l'aval immédiat de la centrale électrique de Gambsheim (67), tandis que l'exemplaire identifié comme appartenant à l'espèce Vimba vimba a été pris à la ligne en Décembre 1989 à deux kilomètres en aval du barrage d'Iffezheim (67) par un pêcheur qui, intrigué par l'aspect de sa capture, l'a confiée au garde-pêche du C.S.P. Un second, pris lui aussi à la ligne dans le même secteur en Octobre 1990, a été identifié mais n'a pas pu être conservé par le garde. 


\section{DESCRIPTION ET IDENTIFICATION}

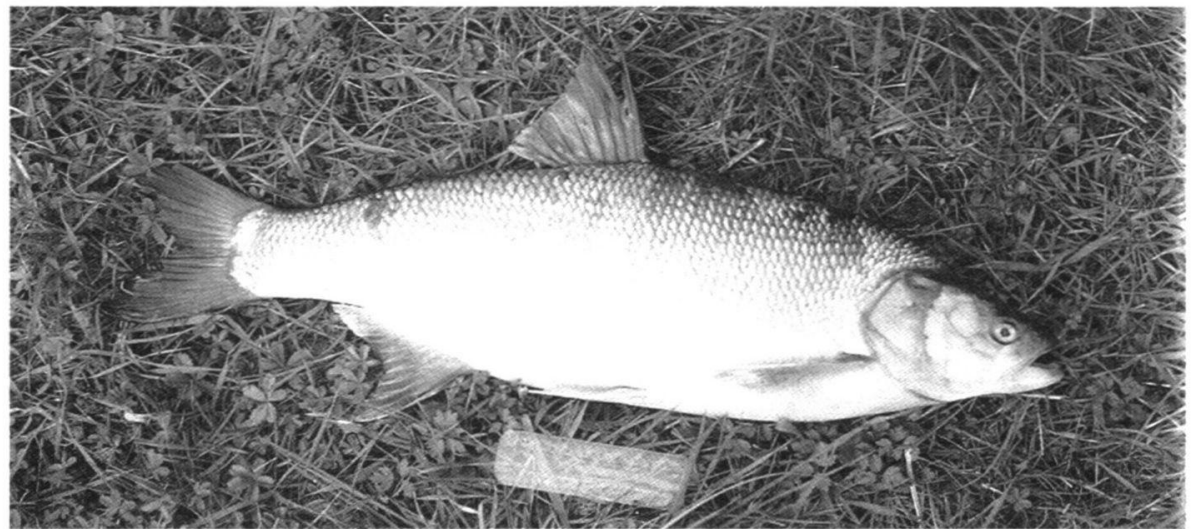

Figure 1 : Exemplaire de l'espèce Aspius aspius capturé dans le Rhin en 1988.

Figure 1 : Individual of the species Aspius aspius captured in the Rhine in 1988.

\section{Aspius aspius}

Le poisson capturé mesure $60 \mathrm{~cm}$ (Iongueur totale) pour un poids de $2.500 \mathrm{~g}$ (Fig. 1) et correspond point par point aux descriptions données par MAITLAND (1977) et BANARESCU (1964). L'Aspe est un cyprinidé susceptible de dépasser $1 \mathrm{~m}$ et $10 \mathrm{~kg}$, caractérisé par une bouche grande (le bord postérieur du maxillaire dépasse l'aplomb du centre de l'œil) et dépourvue de barbillons, un prognathisme inférieur notable, une nageoire dorsale courte et une nageoire anale relativement longue. Tous les décomptes qui ont été effectués sur cet exemplaire ainsi que les proportions calculées (les mensurations étant prises selon les indications de SPILLMANN, 1961) se situent dans les fourchettes indiquées par BANARESCU (1964) (Tableau I), sauf pour le pédoncule caudal qui apparaît particulièrement court sur l'exemplaire étudié ici.

Tableau I : Liste des proportions corporelles et données méristiques de l'exemplaire de Aspius aspius capturé dans le Rhin (1) comparées avec les données disponibles dans BANARESCU (1964) (2). Les longueurs sont exprimées en $\mathrm{mm}$.

Table I : Measurements and meristic counts of the individual Aspius aspius captured in the Rhine (1) compared with the data available in BANARESCU (1964) (2). Lengths are expressed in $\mathrm{mm}$.

\begin{tabular}{|c|c|c|c|c|}
\hline \multirow{2}{*}{ Caractéristiques } & \multicolumn{2}{|c|}{1} & \multicolumn{2}{|c|}{2} \\
\hline & & $\%$ LS & & $\%$ LS \\
\hline Longueur totale & 600 & & & \\
\hline Longueur standard LS & 505 & & & \\
\hline Hauteur maxi du corps & 140 & 27,7 & & $23-28$ \\
\hline Hauteur mini du corps & 50 & 9,9 & & $9,5-11,5$ \\
\hline Longueur du pédoncule caudal & 85 & 16,8 & & $18,5-21,5$ \\
\hline Longueur de la tête $(\mathrm{LT})$ & 121 & 24 & & $22-27$ \\
\hline Longueur du museau & 28 & 5,5 & & $5,5-8,3$ \\
\hline Diamètre de l'œil (DO) & 16 & & & \\
\hline DO/LT (\%) & 13,2 & & $13-17,5$ & \\
\hline Distance prédorsale & 258 & 51 & & $51-55$ \\
\hline Distance préanale & 341 & 67,5 & & \\
\hline Longueur de la dorsale & 59 & 11,7 & & \\
\hline Longueur de l'anale & 82 & 16,2 & & \\
\hline Ecailles ligne latérale (LL) & 73 & & $64-73$ & \\
\hline Ecailles au-dessus de la LL & 11 & & $11-14$ & \\
\hline Ecailles au-dessous de la LL & 6 & & $5-6$ & \\
\hline Rayons de l'anale & III 14 & & || $\mid 12-15$ & \\
\hline Rayons de la dorsale & III 8 & & III $8(9)$ & \\
\hline
\end{tabular}




\section{Vimba vimba}

L'exemplaire étudié mesure $30 \mathrm{~cm}$ pour un poids de $302 \mathrm{~g}$ (Fig. 2) et correspond aussi de façon convaincante aux schémas comme aux descriptions de MAITLAND (1977) et de BANARESCU (1964). Cette espèce possède aussi une nageoire dorsale courte et une anale longue qui, avec un corps comprimé latéralement, contribuent à lui donner une allure de Brème. Elle s'en distingue par un museau particulièrement long qui n'est pas sans rappeler celui du Hotu. La bouche, petite, est très inférieure. Une carène ventrale sans écailles s'étend en avant de l'anus, et une carène dorsale écailleuse caractéristique est présente en arrière de la nageoire dorsale. Les caractères numériques correspondent à ceux qui sont donnés par BANARESCU (1964) (Tableau II).

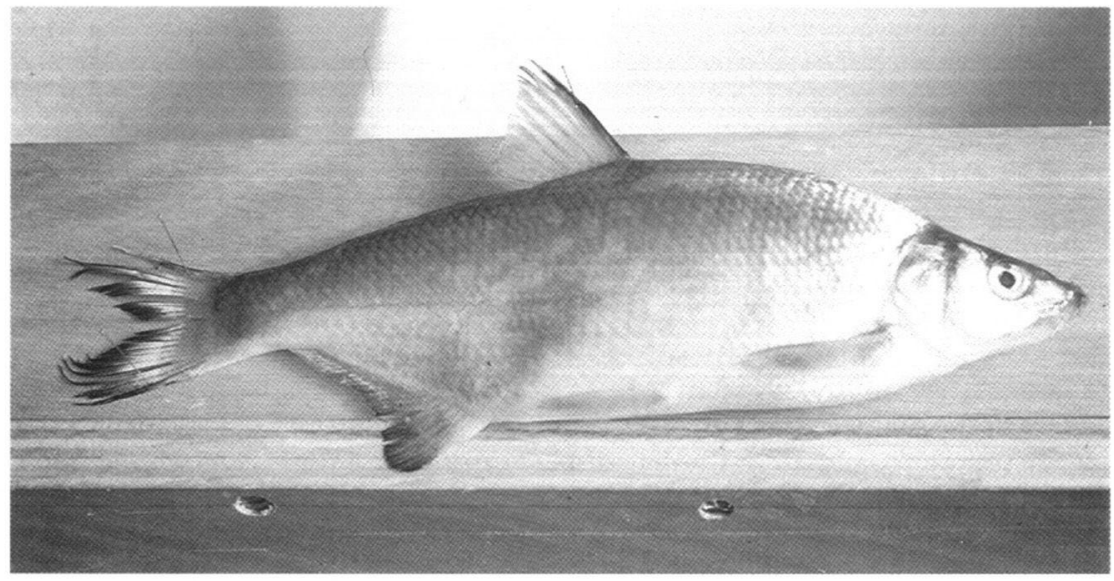

Figure 2 : Exemplaire de l'espèce Vimba vimba capturé dans le Rhin en 1989.

Figure 2 : Individual of the species Vimba vimba captured in the Rhine in 1989.

Tableau II : Liste des proportions corporelles et données méristiques de l'exemplaire de Vimba vimba capturé dans le Rhin (1) comparées avec les valeurs données par BANARESCU (1964) (2). Les longueurs sont exprimées en mm.

Table II : Measurements and meristic counts of the individual Vimba vimba captured in the Rhine (1) compared with the values given by BANARESCU (1964) (2). Lengths are expressed in $\mathrm{mm}$.

\begin{tabular}{|c|c|c|c|c|}
\hline \multirow{2}{*}{ Caractéristiques } & \multicolumn{2}{|c|}{1} & \multicolumn{2}{|c|}{2} \\
\hline & & $\%$ LS & & $\%$ LS \\
\hline Longueur totale & 300 & & & \\
\hline Longueur standard (LS) & 248 & & & \\
\hline Hauteur maxi du corps & 75 & 30,2 & & $23-32,5$ \\
\hline Hauteur mini du corps & 25 & 10 & & $8,3-11,9$ \\
\hline Longueur du pédoncule caudal & 31 & 12,5 & & $12,2-17,9$ \\
\hline Longueur de la tête (LT) & 60 & 24,2 & & $22,8-28,1$ \\
\hline Longueur du museau & 21 & 8,5 & & $6,4-10,1$ \\
\hline Diamètre de l'œil (DO) & 12 & 4,8 & & $4,2-7,3$ \\
\hline $\mathrm{DO} / \mathrm{LT}(\%)$ & 20 & & $20-26$ & \\
\hline Distance prédorsale & 122 & 49,2 & & $48,5-57,3$ \\
\hline Distance préanale & 167 & 67,3 & & $67-73$ \\
\hline Longueur de la dorsale & 28 & 11,3 & & $9,1-14,3$ \\
\hline Longueur de l'anale & 55 & 22 & & $16,3-23,2$ \\
\hline Ecailles ligne latérale (LL) & 59 & & $52-60$ & \\
\hline Ecailles au-dessus de la $L L$ & 10 & & 10 & \\
\hline Ecailles au-dessous de la LL & & & 6 & \\
\hline Rayons de l'anale & III 20 & & III $17-22$ & \\
\hline Rayons de la dorsale & III 9 & & III $8(9)$ & \\
\hline
\end{tabular}




\section{DISCUSSION}

Ces deux espèces qui ne sont mentionnées ni par SPILLMAN (1961), ni dans la liste des espèces françaises de poissons récemment publiée (MINISTĖRE DE L'ENVIRONNEMENT, 1986), appartiennent à un groupe de douze cyprinidés qui sont largement répartis à travers le continent eurasiatique (Abramis, Blicca, Alburnoides, Chondrostoma...). A l'intérieur de celui-ci les genres Vimba et Aspius se caractérisent par une extension limitée vers l'ouest du continent (BANARESCU, 1989). Leurs aires de répartition selon BLANC et al. (1971) sont très superposables, et comprennent dans leurs limites le Caucase, la Russie occidentale, le sud de la Finlande et de la Suède, les PaysBas, l'Allemagne, la Yougoslavie. Le Rhin constitue la limite occidentale de l'aire de répartition de l'Aspe, tandis que celle de Vimba vimba se situe nettement à l'Est de ce fleuve. Cependant les deux espèces y sont signalées comme sporadiques depuis 1976, leur présence étant attribuée à des opérations de repeuplement (LELEK, 1989). Enfin, récemment, Vimba vimba a été signalée pour la première fois en Hollande (CAZEMIER et HEESEN, 1989). Ces captures ne constituent donc pas une surprise, surtout la première, mais méritent néanmoins d'être signalées. La position du Rhin, sa longueur et l'étendue de son bassin en font une voie privilégiée d'extension d'espèces d'Europe Centrale vers l'Ouest, indépendamment des actions humaines éventuelles. La richesse en cyprinidés de l'Europe Centrale et Orientale promet de toutes façons l'apparition en France de nouvelles espèces comme cela s'est souvent produit (ALLARDI, 1984), et a encore été observé récemment (ALLARDI et CHANCEREL, 1988).

Ces deux espèces habitent les cours moyens et inférieurs des fleuves et remontent frayer en eau courante sur des graviers. Vimba vimba peut se rencontrer en eau salée. Si le régime alimentaire de Vimba vimba, qui se nourrit d'invertébrés benthiques, est commun à de nombreuses espèces, celui de l'Aspe est unique parmi les cyprinidés européens. L'adulte est en effet considéré comme presque exclusivement ichthyophage, ce qui, avec la taille qu'il peut atteindre, peut lui conférer un intérêt halieutique tandis que Vimba vimba en apparaît totalement dénué.

S'il n'y a aucun problème pour la désignation de l'Aspe, il n'en est pas de même pour Vimba, espèce pour laquelle peuvent être proposés la Vimbe ou le Serte, traduction phonétique de Zährte, comme cela a été suggéré par SOKOLOV (1989). Bien qu'il ne soit pas question de proposer d'inclure ces espèces dans la liste officielle des espèces françaises, il ne paraît pas inutile de signaler leur existence et de fournir des éléments de diagnose aux agents susceptibles d'avoir à les déterminer, en particulier les gardes-pêche.

\section{BIBLIOGRAPHIE}

ALLARDI J., 1984. Introduction et acclimatation des poissons d'eau douce en France. Historique et bilan. EIFAC Techn. Paper, 42-2, 427-435.

ALLARDI J. et CHANCEREL F., 1988. Sur la présence en France de Pseudorasbora parva (Schlegel, 1842). Bull. Fr. Pêche Pisc., 308, 35-37.

BANARESCU P., 1964. Fauna Republicii Populare Romine. Pisces Osteichthys XIII, 559 p.

BANARESCU P., 1989. Zoogeography and history of the freshwater fish fauna of Europe. In : The freshwater fishes of Europe, Vol. 1 II, Ed. Aula Verlag, Wiesbaden, 88-103.

BLANC M., BANARESCU P., GAUDET J.L., HUREAU J.C., 1971. Poissons des eaux continentales d'Europe, catalogue multilingue. Fishing News (books).

CAZEMIER W.G., HEESEN M.J., 1989. First record of Vimba vimba (Linnaeus 1758) (Pisces Cyprinidae) in the Nederlands. Bull. Zool. Museum, 12 (6), 97-100.

LELEK A., 1989. The Rhine river and some of its tributaries under human impact in the last two centuries. In International large river symposium (LARS), Ed. D.P. Dodge, 504514.

MAITLAND P.S., 1977. Les poissons des lacs et rivières d'Europe en couleurs. Elsevier Séquoia, $256 \mathrm{p}$. 
MINISTĖRE DE L'ENVIRONNEMENT, 1986. Arrêté du 17 décembre 1985 fixant la liste des espèces de poissons, de crustacés et de grenouilles représentées dans les eaux visées à l'article 413 du Code rural. Journal officiel de la République Française, 26 janvier 1986.

SOKOLOV V.E., 1989. Dictionnaire des noms des animaux en cinq langues. Poissons. Russky Yazik Publishers. Moscou. 733 p.

SPILLMANN C.J., 1961. Faune de France. Poissons d'eau douce. Editions Paul Lechevalier, Paris $303 \mathrm{p}$. 